\title{
ESTUDO FILOGENÉTICO DE MESEMBRINELLINAE COM ÊNFASE NO GÊNERO EUMESEMBRINELLA TOWNSEND (DIPTERA, CALLIPHORIDAE) ${ }^{1}$
}

\author{
Ronaldo Toma ${ }^{2}$ \\ Claudio José Barros de Carvalho ${ }^{2}$
}

\begin{abstract}
PHYLOgENETIC STUDY OF MESEMBRINELINAE, WTTH EMPHASIS IN EUMESEmbrinella TOWNSEnd (DiPTERA, CAlliphoridaE). A study of phylogenetic relationships of the genera of Mesembrinellinae and of the species of Eumesembrinella Townsend, 1931, on the ground of shared derived character states (synapomorphies) is presented. Resulting the following assumption: Laneella Mello, 1967 presenting sister group relationship with all other Mesembrinellinae, followed by Souzalopesiella Guimarães, 1977, M. peregrina Aldrich, 1922 that was isolated from Mesembrinella Giglio-Tos, 1893. Mesembrinella was considered paraphiletic, its species were judged in changeable positions at the bifurcation point of analysis of Albuquerquea Mello, 1967 with the tricotomic interrelationships of Thompsoniella Guimarães, 1977, Huascaromusca Townsend, 1918 and Eumesembrinella Townsend, 1931. The phylogeny of the genus Eumesembrinella shows E. cyaneicincta in basal position, followed by $E$. quadrilineata that showed sister group relationship with $E$. randa plus E. benoisti. Mesembrinella currani Guimarães, 1977 and Eumesembrinella benoisti (SÉGUY 1925) were revalided.
\end{abstract}

KEY WORDS. Calliphoridae, Mesembrinellinae, Eumesembrinella, classification, phylogeny

Este grupo exclusivamente neotropical vem sendo estudado por vários pesquisadores (ALDRICH 1922; TOWNSEND 1931, 1935; HALL 1948; MELLO 1967; GuIMARÃes 1977; PERIS \& MARILUIS 1984). No entanto, a maioria dos trabalhos realizados ficou restrito apenas aos propósitos de descrição e identificação. Excetuando GUIMARÃES (1977), podemos afirmar praticamente que quase nada foi postulado a respeito das relações filogenéticas intergenéricas para Mesembrinellinae ou interespecíficas para Eumesembrinella Townsend, 1931. No entanto, GUIMARÃES (1977) utilizou a metodologia tradicional, possibilitando a formação de agrupamentos artificiais, grupos parafiléticos. Os objetivos deste trabalho são: 1) estabelecer um esboço das relações intergenéricas de parentesco para Mesembrinellinae e 2) uma hipótese de parentesco interespecífico para Eumesembrinella, através do método cladístico.

1) Contribuição número 814 do Departamento de Zoologia, Universidade Federal do Paraná.

2) Departamento de Zoologia, Universidade Federal do Paraná, Caixa Postal 19020,

81531-990 Curitiba, Paraná, Brasil. 


\section{MATERIAL E MÉTODOS}

O material estudado pertence as seguintes instituições: DZUP - Coleção do Departamento de Zoologia da Universidade Federal do Paraná, Departamento de Zoologia, Curitiba, Brasil; INPA - Instituto Nacional de Pesquisa da Amazônia, Manaus, Amazonas, Brasil; MZSP - Museu de Zoologia da Universidade de São Paulo, São Paulo, Brasil.

Foram examinados 2.472 exemplares. Incluem-se, neste total, 16 das 30 espécies nominais reconhecidas por GUIMARÃES (1977), com representação de todos os gêneros. Foram examinadas as seguintes espécies de Mesembrinellinae: Mesembrinella bellardiana Aldrich, 1922; M. peregrina Aldrich, 1922; M. batesi Aldrich, 1922; M. semihyalina Mello, 1967; M. currani Guimarães, 1977; M. bicolor (Fabricius, 1805); Albuquerquea latifrons Mello, 1967; Eumesembrinella quadrilineata (Fabricius, 1805); E. benoisti (Séguy, 1925); E. cyaneicincta (Surcouf, 1919); E. randa (Walker, 1849); Huascaromusca aeneiventris (Wiedemann, 1830); H. purpurata (Aldrich, 1922); Thompsoniella anomala Guimarães, 1977; Souzalopesiella facialis (Aldrich, 1922); Laneella nigripes Guimarães, 1977. Foram também examinadas as seguintes espécies externas a Mesembrinellinae, pertencentes à Calliphoridae, utilizadas para comparação: Toxotarsinae, Sarconesia chlorogaster (Wiedemann, 1830); Calliphorinae, Calliphora vicina Robineau-Desvoidy, 1830; Helicoboscinae, Helicobosca palpalis (Robineau-Desvoidy, 1830); Chrysomyinae, Chrysomyia albiceps (Wiedemann, 1830); Hemilucilia semidiaphana (Rondani, 1850), Paralucilia adespota James, 1985, Chloroprocta idioidea (Robineau-Desvoidy, 1830) e Cochliomyia macellaria (Fabricius, 1775).

Os termos morfológicos aqui empregados foram baseados em MCALPINE (1981).

O estabelecimento do parentesco filogenético foi feito através do método cladístico. Metodologia criada por HENNIG (1965 1966) e desenvolvida posteriormente por vários pesquisadores (ELDREDGE \& CRACRAFT 1980; NELSON \& PLATNICK 1981; WILEY 1981).

\section{RESULTADOS E DISCUSSÃO}

Mesembrinellinae tem sido mais frequentemente associado como um grupo subordinado de Calliphoridae (SHANNON 1923; HALL 1948; ROBACK 1951; Mello 1967; JAMES 1970; HeNNIG 1973). HALL (1948) considerou o grupo como uma aberração entre os Calliphoridae; o hábito reprodutivo, as estruturas dos orgãos reprodutivos e certos detalhes da anatomia externa das fêmeas são totalmente diferentes dos Calliphoridae típicos. No entanto, baseando-se em alguns caracteres externos dos adultos e genitália do macho, o autor concluiu que Mesembrinellinae pertence aos Calliphoridae.

GUIMARÃES (1977), inspirado por CROSSKEY (1965) e baseado em caracteres listados por HaLl (1948) para separar Mesembrinellinae das demais subfamílias de Calliphoridae, elevou aquela à mesma categoria da última, colocandoas como grupos estreitamente relacionados. 
ROGNES (1986), através de estudos filogenéticos, afirmou que Mesembrinellinae possui todas as apomorfias de Calliphoridae listadas em seu trabalho (adultos com as superfícies torácicas do pró-esterno, pró-episterno, parede pós-alar e basisterno com cerdas, adultos machos com a terminação distal de cada processo dorsolateral (parafalo) do aedeago bem esclerotinizado, livre do distifalo por uma considerável distância e figurado como um processo grande e estreito, com placas em ganchos ou denteadas ou nuas), devendo aquele táxon permanecer como subgrupo de Calliphoridae.

Embora os estados plesiomórficos de caráter para Mesembrinellinae não sirvam para sustentar relação de parentesco, não obstante, não contradizem também proximidades de descendentes, podendo ser um forte indicador de parentesco em pontos de análise mais amplos.

GUIMARÃES (1977) utilizou a macrolarviparidade, subescutelo desenvolvido e ovipositor não telescopado, para excluir Mesembrinellinae dos Calliphoridae e para a sustentar seu status novo. No entanto, estas condições devem ser avaliadas em níveis mais amplos.

O desenvolvimento da macrolarviparidade foi correlacionado com aumento da atividade secretora da espermateca, sugerindo um desvio de sua adaptação primária de armazenamento e provisão de nutrientes para os espermatozóide para o de nutrição da larva. Para a utilização deste estado na sustentação da hipótese de monofilia do grupo, deve ser levado em conta a sua ocorrência em outros grupos subordinados dentro de Calliphoridae (Helicoboscinae, Ameniinae e Phumosiniinae). Talvez estes grupos formem um agrupamento monofilético com Mesembrinellinae (K. Rognes, com. pessoal).

Subscutelo desenvolvido (Fig. 2) é um estado considerado autapomórfico para Tachinidae (ROGNES 1986), embora também seja encontrado em alguns grupos de Oestroidea: Oestridae (Oestrinae), Calliphoridae (Ameniinae e Mesembrinellinae). Com interpretação do aparecimento deste estado independentemente nos grandes grupos de Oestroidea, pode-se levantar a hipótese de uma relação de grupo-irmão entre Ameniinae e Mesembrinellinae.

O ovipositor do tipo telescopado é uma condição que ocorre desde os Brachycera Orthorrapha e acentua-se nos Muscomorpha (MCAlPINE 1981). Baseado neste relato e na evidência de ocorrência da condição não telescopada também Ameniinae (CROSSKEY 1965), pode-se sugerir um reforço na idéia de monofilia entre este táxon e Mesembrinellinae. No entanto, maiores estudos morfológicos e biogeográficos devem ser feitos.

A lista abaixo refere-se aos estados de caráter que delatam as monofilias dos grupos. O sinal de $\left(^{*}\right)$ indica que o estado é homoplásico. A listagem dos estados de caráter deste trabalho foram retiradas de literatura, principalmente, GuimarĀEs (1977), e de observações feitas pelo autor senior.

Mesembrinellinae forma um agrupamento monofilético, apesar da diversificação dos gêneros que o compõem. A monofilia do grupo é estabelecida pelas supostas sinapomorfias distribuídas entre seus gêneros (Fig. 1): 


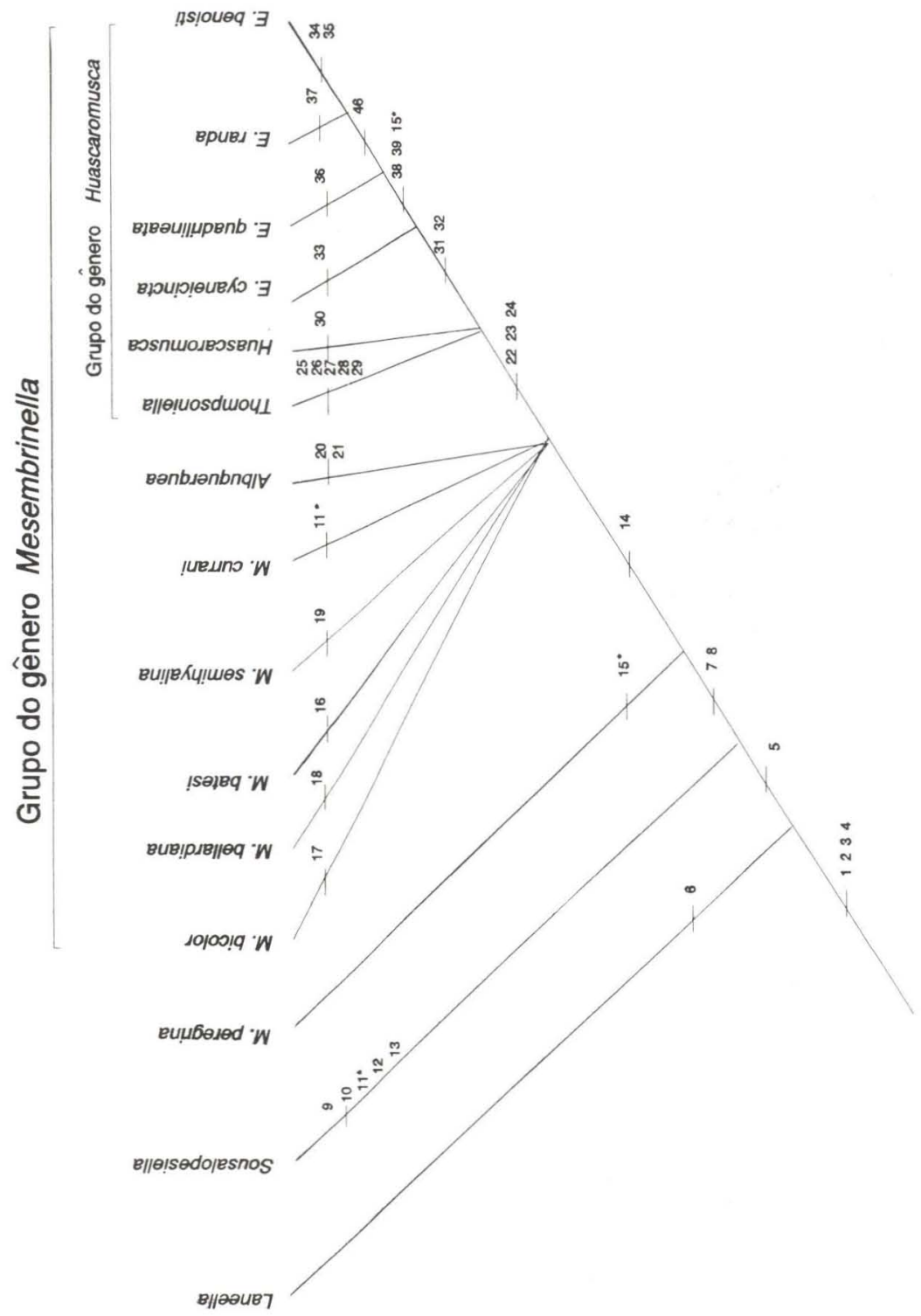

Fig. 1. Cladograma intergenérico de Mesembrinellinae e interespécifico de Eumesembrinella. Caracteres são apresentados e discutidos no texio.

1 - Espiráculo metatorácico único, reniforme, com abertura dorsal (Fig. 2).

PAPE (1992) frisou a dificuldade de comparar esta condição com o estado bilobado do espiráculo metatorácico. Esse estado de caráter é único com respeito ao plano básico dos Calliphoridae (GUIMARĀES 1977). O estado primitivo apre- 
senta-se sob a forma bilobada, podendo variar tanto a forma e tamanho dos lobos anterior e posterior quanto a posição da abertura do espiráculo, dependendo da espécie.

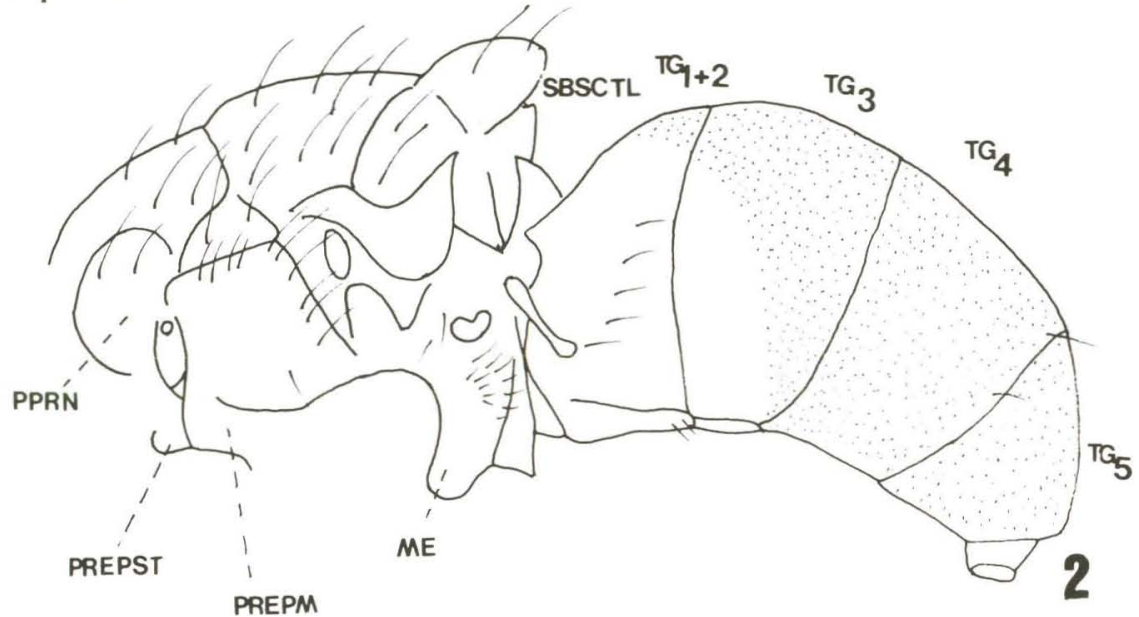

ACR S

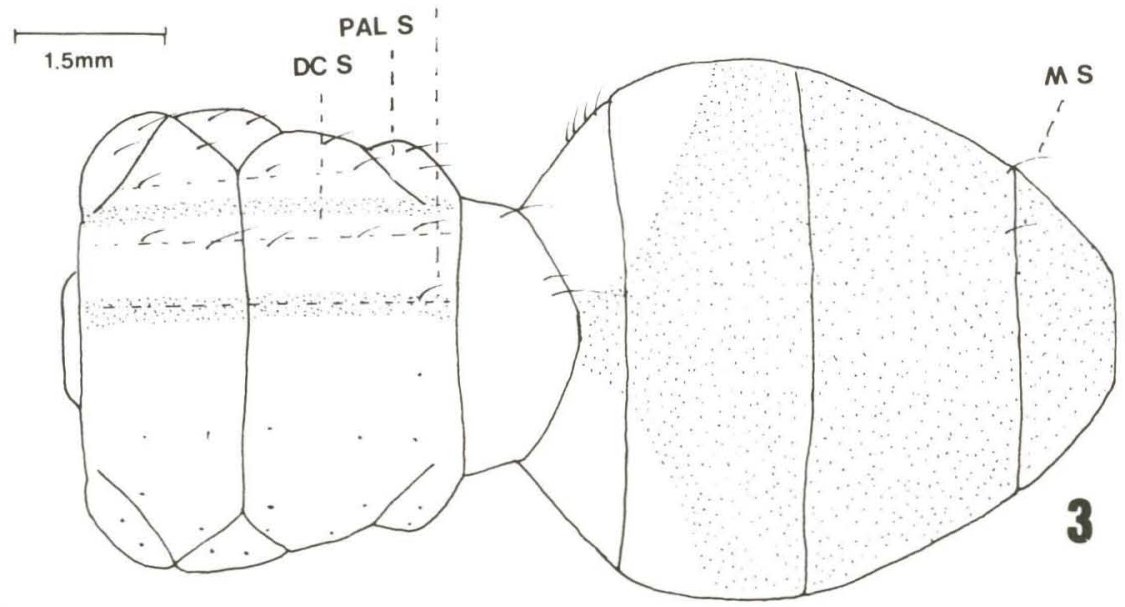

Figs 2-3. Eumesembrinella quadrilineata. (2) Vista lateral; (3) vista dorsal. Abreviaturas: (mr) mero; (pprn) pós-pronoto; (prepm) pró-epimero; (prepst) pró-episterno; (sbsctl) subescutelo; (tg) tergito; (acrs) cerda acrostical; (de s) cerda dorsocentral; (m s) cerda marginal; (pprn s) cerda pós-pronotal; (pal s) cerda pós-alar.

2 - Espermateca alongada e esclerotinizada.

Este padrão é único dentro dos Calliphoridae, sendo que a forma primitiva ocorre sob as formas ovóide e sub-cilíndrica. 
3 - Asa com mácula na porção médio-apical, próximo as veias Radio1 e Radio2 +3 (Fig. 4).

Este estado de caráter é único dentro dos Calliphoridae, sendo sua presença em Mesembrinellinae considerada sinapomórfica para suas espécies.

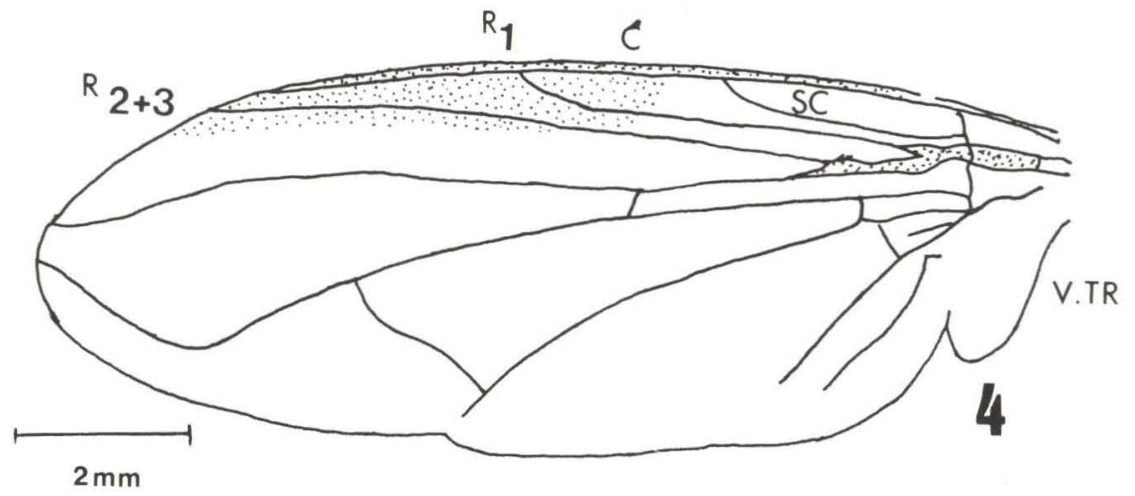

Fig. 4. Asa de Eumesembrinella quadrilineata. Abreviaturas: (C) costa; (Sc) Subcosta; (R $\mathbf{R}_{1}$ Radial 1; $\left(\mathbf{R}_{2+3}\right)$ Radial 2+3; (v.tr) veia tronco.

4 - Esternito 8 ausente na fềmea (Fig. 8)

Todos os Calliphoridae examinados, excetuando Mesembrinellinae, apresentaram o esternito 8. A ausência deste esclerito ocorreu na maioria dos Mesembrinellinae, exceto em Thompsoniella. A ausência do esternito 8 foi interpretado como sinapomórfico para todas as espécies de Mesembrinellinae e sua presença em Thompsoniella foi considerada como um ganho secundário. Esta questão será discutida posteriormente.

Embora a monofilia de Mesembrinellinae esteja relativamente bem fundamentada, o mesmo não pode ser dito para os seus grupos subordinados.

GUIMARÃES (1977) propôs uma classificação supragenérica, baseado na espermateca, quetotaxia (principalmente do tórax) e esclerito cefalofaringeal da larva I.

Apresentou a espermateca sob três padrões básicos: tipo $\mathrm{A}=$ cápsula em forma "salsichóide", marrom, alongada, envolta por uma camada espessa de células grandes e vacuoladas (Fig. 5); tipo $\mathrm{B}=$ cápsula modificada em um longo tubo filiforme, bastante dilatada na base, transversalmente corrugada e forrada por uma única camada de células vacuoladas (Fig. 6) e tipo $\mathrm{C}=$ assemelha-se muito a anterior, diferindo-se apenas por ter a base mais delgada (Fig. 7). Através destes padrões, GuIMARÃES (1977) dividiu sua família Mesembrinellidae em três subfamilias: Laneellinae possuindo o tipo A, composta pelo gênero monoespecífico Laneella; Souzalopesiellinae apresentando o tipo B, constituida também por um gênero monoespecífico, Souzalopesiella, e Mesembrinellinae possuindo o tipo C e sendo composta pelos demais gêneros. Apesar desta separação em três grupos, não fez menção sobre hipótese explícita a respeito do parentesco. 
Um estudo mais detalhado da espermateca oferece uma hipótese sobre a monofilia de Souzalopesiella + Mesembrinella + Albuquerquea + Huascaromusca +Thompsoniella +Eumesembrinella (Fig. 1).
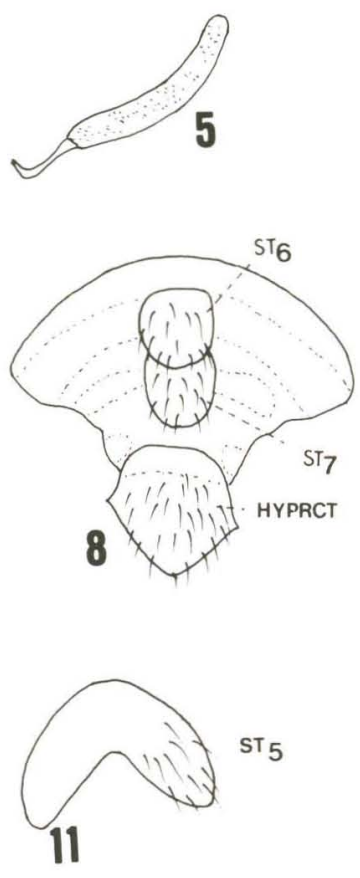

ST 5

$\mathrm{si}_{5}$
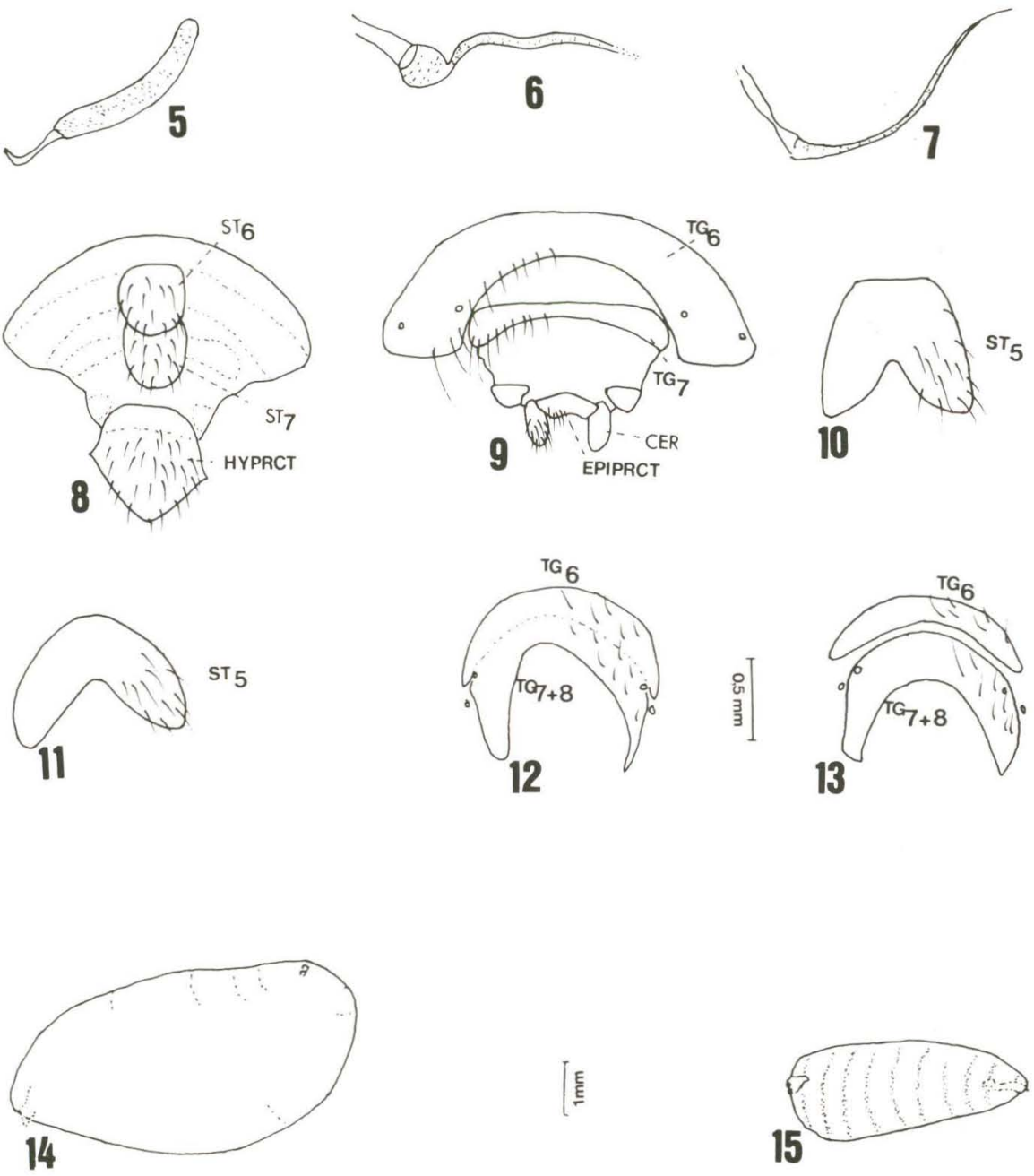

Figs 5-15. (5) Laneella nigripes, espermateca tipo A; (6) Souzalopesiella facialis, espermateca tipo B; (7) Eumesembrinella quadrilineata, espermateca tipo C; (8) terminália da fêmea de E. quadrilineata, vista ventral: (9) terminália da fêmea de E. quadrilineata, vista dorsal; (10) E. quadrilineata, esternito 5 do macho; (11) Laneella nigripes, esternito 5 do macho; (12) E. quadrilineata, tergito 6 e sintergito $7+8$ do macho; (13) L. nigripes, tergito 6 e sintergito $7+8$ do macho; (14) E. cyaneicincta, larva; (15) L. nigripes, larva. Abreviaturas: (epipret) epiprocto; (st) esternito; (hyprct) hipoprocto; (tg) tergito. 


\section{Condição sinapomórfica:}

5 - Afilamento da porção apical da espermateca (tipo B e tipo C).

Partindo da forma ovóide primitiva encontrada fora de Mesembrinellinae, a interpretação mais parcimoniosa da polarização dos estados da espermateca presentes dentro do grupo compreende três passos: 1) um alongamento inicial de seu comprimento, que pode ser representado pela espermateca do tipo A, encontrada em Laneella; 2) seguido de um afilamento de sua porção apical, dando uma espermateca em forma de capuz, do tipo B e C, encontrada nos demais gêneros e 3) por último um estreitamento basal, configurando uma espermateca do tipo $\mathrm{C}$, encontrada em Mesembrinella, Albuquerquea, Huascaromusca, Thompsoniella e Eumesembrinella.

Baseando-se nesta interpretação de polarização, o alongamento inicial do comprimento da espermeteca, espermateca do tipo A, indica uma sinapomorfia para os Mesembrinellinae. O posterior afilamento de sua porção apical, espermatecas dos tipos B e C, supõe uma sinapomorfia para Souzalopesiella, Mesembrinella, Albuquerquea, Huascaromusca, Thompsoniella e Eumesembrinella, sendo que, neste ponto de análise, a espermateca do tipo A, que ocorre em Laneella, é tida como primitiva (Fig. 5). O último passo, o estreitamento da porção basal da espermateca, é uma novidade evolutiva para Mesembrinella, Albuquerquea, Huascaromusca, Thompsoniella e Eumesembrinella, sendo interpretada a espermateca do tipo B, neste ponto de análise, como uma condição primitiva (Fig. 6).

\section{MCNOFILIA DE LANEELLA}

6 - Padrão de coloração das garras tarsais

A monofilia de Laneella pode ser suportada pela evidência de um padrão de coloração encontrado nas garras tarsais: garras com bases brancas e ápices escuros. Nos demais Mesembrinellinae o padrão de cor encontrado são as bases alaranjadas e os ápices marrom-escuros. Nos Calliphoridae examinados o padrão variou, porém nenhum se assemelhou ao encontrado em Laneella.

Monofilia de Mesembrinella + Albuquerquea + Huascaromusca + Thompsoniella + Eumesembrinella (Fig. 1). A monofilia deste grupo é concordante com a delimitação da subfamília Mesembrinellinae de GUIMARÃES (1977).

\section{Condições sinapomórficas para os gêneros do grupo:}

7 - Larva ovóide (Fig. 14).

GUIMARĀES (1977) fez comentário sobre o compartilhamento entre Laneellla e Souzalopsiella de certos estados de caráter como: larvas com padrão de coloração branco-acinzentada e formato do corpo cônico-cilíndrico (Fig. 15), contra o padrão de coloração alaranjado e o formato ovóide nos demais gêneros. No entanto, para uma hipótese de relação de parentesco destes dois gêneros baseada nestas condições, é preciso avaliar como ocorre a distribuição destes estados de caráter fora do grupo. O padrão de coloração da larva é variado, enquanto o formato geral do corpo é cônico-cilíndrico, nos Calliphoridae (SHEWELL 1987). 
Conclui-se que a aproximação destes dois gêneros através destes estados de caráter é artificial, devido ao não compartilhamento único destas condições. Por outro lado, a ocorrência de larvas com o formato ovóide nos demais taxons aparece como um estado de carater único, sustentando a monofilia deste grupo.

8 - Estreitamento da porção basal da espermateca (espermateca do tipo C).

Já foi comentado anteriormente na discussão do caráter espermateca e é concordante com a hipótese acima. Souzalopesiella ocupa junto a Laneella uma posição basal entre os Mesembrinellinae. A monofilia desse gênero monoespecífico pode ser suportada pelos seguintes estados autapomórficos, tomando como análise Mesembrinellinae:

9 - Cercos fortemente recurvados, alongados e delgados em vista lateral.

A condição presente nos Mesembrinellinae é forma robusta, não fortemente recurvada como a anterior, quando visto lateralmente.

10 - Esternitos abdominais grandes e subquadrangulares.

Os demais Mesembrinellinae apresentam os esternitos abdominais sob a forma oval, o mesmo ocorrendo nos Calliphoridae examinados.

$11^{*}$ - Cerdas dorsocentrais 3:3.

Com exceção de Thompsoniella, que possui o padrão 2:2, a subfamília apresenta um arranjo uniforme de dorsocentrais 2:3 (Fig. 3), sendo o número de cerdas dorsocentrais 3:3 autapomórfico para Souzalopesiella.

12 - Cerdas acrosticais 3:2.

O número padrão da subfamília é 2:3, sendo a condição $3: 3$ considerada derivada.

13 - Espiráculos abdominais direitos 6 e 7 unidos, no tergito 6 do macho.

Todos os Mesembrinellinae estudados apresentaram os espiráculos abdominais 6 e 7, direitos e esquerdos, separados. Esta mesma condiçãos foi evidênciada nos Calliphoridae examinados (Fig. 12 e 13).

Possivelmente a reunião das espécies contidas no gênero Mesembrinella seja um agrupamento não-monofilético. Nenhum estado derivado de caráter que sustente a monofilia deste táxon foi reportado. Este grupo tem sido reconhecido através da combinação de diversos estados de caráter, como se pode notar na diagnose dada por GUIMARÃEs (1977):

"Mesembrinellinae com as seguintes combinações: abdome marrom com reflexo variando do azul ao verde, acrosticais 2:2, dorsocentrais 2:3, póspospronotais presentes, pospronoto (calo umeral) com três cerdas distintas, discais no tergito 5 ausentes. Larva I com integumento sem espinhos ou placas esclerotinizadas; esclerito labial grande e espatulado, arredondado no ápice; esclerito hipostomal não fusionado com o labial. Aedeago com processo dorsolateral não fusionado ao processo ventrolateral (hipofalo)."

Como consequência do tamanho do táxon (14 espécies nominais) e a 
possessão de uma relativa diversidade estrutural, a maioria dos estados de caráter apresentado na diagnose varia bastante entre as espécies contidas neste gênero e/ou faz parte de um plano mais amplo.

14 - Fusionamento do sintergito $7+8$ ao tergito 6 nos machos.

Uma forte evidência da parafilia de Mesembrinella é proporcionada ao analisarmos o caráter relacionado com fusionamento do sintergito $7+8$ ao tergito 6 nos machos. Este caráter apresenta-se basicamente sob três condições: o sintergito $7+8$ ocorrer separado do tergito 6 (Fig. 13) por de uma estreita membrana; pode se apresentar parcialmente fusionado ao tergito 6, ou apresentarse fusionado a este (Fig. 12). Por enquanto os dois últimos serão tomados como um só.

O fusionamento do sintergito $7+8$ ao tergito 6 foi considerado derivado, evidenciando que a condição não fusionada ocorreu na maioria dos Calliphoridae examinados, exceto em Chrysomyia albiceps, e parece ocorrer em grupos fora de Calliphoridae, como, por exemplo, em Muscidae, comparação realizada através de figuras (HUCKETT \& VOCKEROTH 1987). Das espécies de Mesembrinella estudadas ( $M$. bellardiana, $M$. peregrina, $M$. batesi, $M$. semihyalina, $M$. currani e $M$. bicolor), a maioria apresentou o fusionamento do sintergito $7+8$ ao tergito 6 , condição que só não é encontrada em $M$. peregrina. Nos demais gêneros, a condição derivada esteve presente em Albuquerquea, Huascaromusca, Thompsoniella e Eumesembrinella, enquanto a primitiva ocorreu em Laneella e Souzalopesiella. Não contrariando a idéia de basalidade destes dois gêneros, dentro da subfamília.

No presente estudo, nenhum estado apomórfico foi hipotetizado para suportar a monofilia entre as espécies de Mesembrinella que apresentaram a condição derivada de fusionamento. Cada ramo foi definido através de suas autapomorfias específicas. O mesmo aconteceu com o ramo anterior à bifurcação, com $M$. peregrina. É possível que num estudo posterior, incluindo todas as espécies de Mesembrinella, haja a formação de diversos grupos genéricos independentes, no ponto anterior e posterior definido pelo estado de caráter 14 .

MONOFILIA DAS ESPÉCIES DE MESEMBRINELLA (Fig. 1):

\section{Mesembrinella peregrina}

\section{Condição autapomórfica:}

$15^{*}$ - Ápice do processo dorsolateral do edeago com dentes (Fig. 16)

Apesar da presença deste estado em algumas espécies de Eumesembrinella, podemos hipotetizar a evolução independente desta condição em ambas as linhagens. Esta questão será discutida posteriormente. 

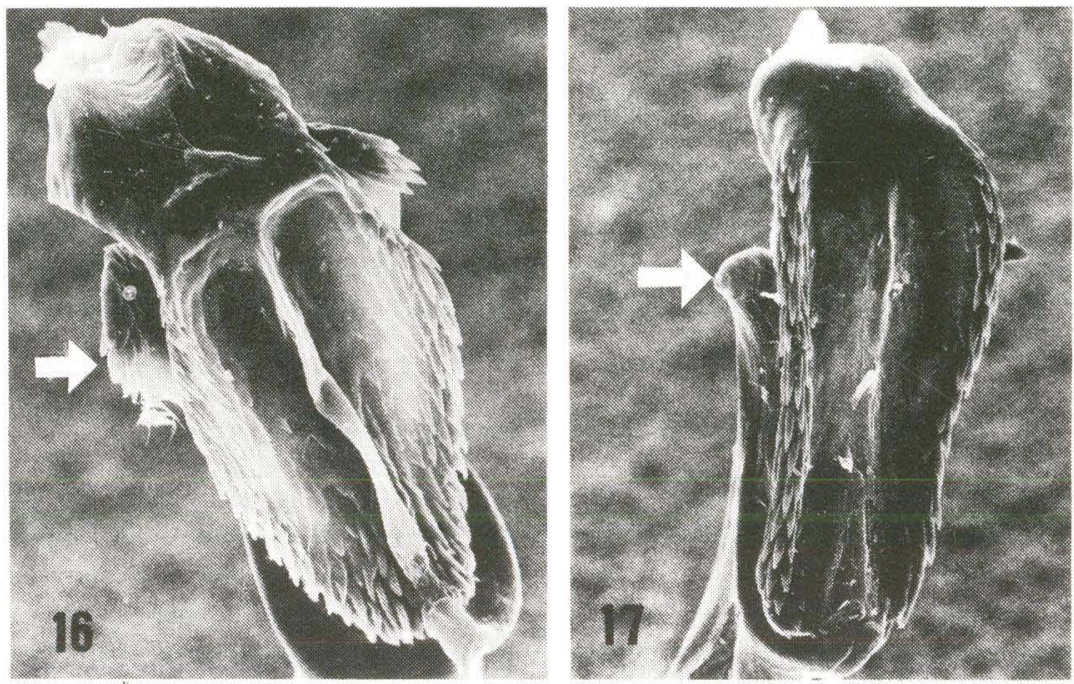

Figs 16-17. Vista ventral do processo dorsolateral do aedeago. (16) E. quadrilineata, aumento de 302 vezes; (17) E. cyaneicincta.

\section{Mesembrinella batesi}

\section{Condição autapomórfica:}

16 - Abdome com intensa polinosidade.

Apesar da ocorrência de polinosidade abdominal na maioria das espécies de Mesembrinellinae, nenhuma delas apresentou uma condição tão intensa quanto M. batesi, sendo esta condição considerada derivada.

\section{Mesembrinella bicolor}

\section{Condição autapomórfica:}

17 - Ausência do padrão de mancha na parte distal da asa, próximo as veia Radio1 e Radio2 +3 .

Com relação a Mesembrinellinae, este padrão de mancha está presente na maioria das espécies (Fig. 4), exceto em M. bicolor e Eumesembrinella benoisti. No entanto, pode-se afirmar que esta perda se deu independentemente.

\section{Mesembrinella bellardiana}

\section{Condição autapomórfica:}

18 - Acrosticais 2:2.

O número típico de cerdas acrosticais em Mesembrinellinae é 2:3, sendo a condição 2:2 considerada derivada. 


\section{Mesembrinella semihyalina}

\section{Condição autapomórfica:}

19- Manchas ao redor das veias na porção médio apical da asa.

Dentre as espécies estudadas neste trabalho, nenhuma apresentou estado semelhante. Não obstante, existe na literatura (GUIMARÃES 1977) descrições similares a este padrão em $M$. umbrosa Aldrich, 1922. Tal coincidênca deve ser melhor avaliada. Por enquanto, a interpretação deste estado será como autapomórfico para $M$. semihyalina.

\section{Mesembrinella currani Guimarães, 1977, sp.rev.}

\section{Condição autapomórfica:}

As cerdas dorsocentrais 3:3 (estado de caráter $11 *$ ) ocorrem em $M$. currani e Souzalopesiella. No entanto, o compartihamento desta condição por estes dois taxons acarreta uma hipótese menos parcimoniosa, com três hipóteses ad hoc de homoplasias (caráteres 7, 8 e 14), do que a sustentação de uma hipótese de evolução independente.

M. currani foi colocada em sinonímia de $M$. umbrosa por PERIS \& Mariluis (1984). No entanto, a descrição dada por GuIMARÃes (1977) para $M$. umbrosa não confere com o holótipo de $M$. currani estudado neste trabalho. Sigo por esta evidência manter separadas ambas as espécies.

Monofilia de Albuquerouea (Fig. 1).

\section{Condições autapomórficas:}

20 - Machos dicópticos.

A tendência à condição holóptica está normalmente restrita aos machos, onde é associada ao acasalamento aéreo (MCALPINE \& MUNROE 1968). Esta condição está presente na maioria dos Mesembrinellinae, exceto em Albuquerquea, e também ocorre em todos os demais Calliphoridae estudados, com exceção de Sarconesia chlorogaster. A presença da condição dicóptica nesta espécie e em Albuquerquea é melhor interpretada como evolução independente.

21 - Macho com cerdas interfrontais.

Nenhum macho de Mesembrinellinae, exceto Albuquerquea, nem dos demais Calliphoridae examinados apresentou esta condição, sendo esta considerada derivada para o gênero.

Monofilia de Thompsoniella + Huascaromusca+Eumesembrinella. Estados de caráter sinapomórfico para estes taxons, com respeito aos Mesembrinellinae:

22 - Cerdas acrosticais 0:1 (Fig. 3).

GUIMARÃES (1977), baseado no número típico de cerdas acrosticais 2:3 encontrado na sua família Mesembrinellidae, classificou os três gêneros dentro de 
Eumesembrinellina. A utilização do padrão de cerdas acrosticais 0: 1 na delimitação destes três gêneros é corroborada pelas duas hipóteses seguintes:

23 - Cerdas pospronotais 2 (Fig. 3).

O número predominante de cerdas pospronotais em Mesembrinellinae é 3 , sendo este o número encontrado em todos os demais Calliphoridae examinados. Baseado nesta evidência, podemos concluir que a presença de 2 cerdas pospronotais é uma sinapomorfia para Thompsoniella, Huascaromusca e Eumesembrinella.

24 - Fêmures médio e posterior com mácula na porção distal.

Este estado de caráter ocorre em Thompsoniella, Huascaromusca e Eumesembrinella cyaneicincta. A não ocorrência desta condição em todas as espécies de Eumesembrinella é mais parcimoniosamente explicada como uma perda secundária deste estado. A hipótese de monofilia de Thompsoniella, Huascromusca e E. cyaneicincta, requer hipóteses ad hoc de homoplasia provenientes dos estados de caráter que suportam a monotilia de Eumesembrinella, que serão comentadas mais adiante.

\section{MonofiLIA de THOMPSONIELla (Fig. 1)}

\section{Condições autapomórficas:}

25 - Tergito abdominal 5 duas vezes o comprimento do tergito 4.

Esta condição usada por GUIMARÃES (1977) na diagnose do gênero é única entre os Mesembrinellinae, onde o abdome apresenta-se robusto, convexamente arredondado e moderadamente uniforme em cada gênero (Fig. 3).

26 - Edeago com epifalo curvado apicalmente.

Nos Mesembrinellinae o epifalo possui a base dilatada, afilando gradualmente em direção ao ápice e sem curvatura a este nível. Em Thompsoniella, o epifalo apresenta a largura mais ou menos homogênea em todo o seu comprimento e forte curvatura apical, sendo esta condição considerada derivada.

27 - Dorsocentrais 2:2.

O padrão predominante de dorsocentrais em Mesembrinellinae é 2:3. Sendo o padrão 2:2 considerado autapomórfico para Thompsoniella.

28 - Hipoprocto mais longo que largo.

Hipoprocto largo, com a margem posterior formando ângulo, margem anterior arredondada e saliências laterais (Fig. 8) aparece mais ou menos homogêneo na maioria das espécies de Mesembrinellinae, exceto em Thompsoniella. Nesta espécie o hipoprocto é mais longo que largo, sendo considerada condição autapomórfica

29 - Presença do esternito 8 e ausência do tergito 8.

Estes dois caracteres são discutidos conjuntamente. A ausência do esternito 8 foi assinalada como uma sinapomorfia na definição da monofilia de Mesembrinellinae. Sua presença em Thompsoniella é melhor explicada como um 
ganho secundário. A hipótese de plesiomorfia para este estado de caráter em Thompsoniella requer várias hipóteses ad hoc de homoplasias, ou seja, todas as sinapomorfias que estão definindo os ramos à direita do cladograma a partir do estado de caráter 4 (ausência do tergito 8) (Fig. 1).

A ausência de um esclerito na posição do tergito 8 sugere duas hipóteses: ausência pela perda do tergito 8 , tendo como consequência a admissão de mais uma autapomorfia e a aceitação da presença do esternito 8 como homoplásica com relação as espécies de Calliphoridae externas a Mesembrinellinae, ou ausência pela migração do tergito 8 para a porção ventral do abdome, tendo como consequência uma autapomorfia e a perda da hipótese de homoplasia. Do ponto de vista teórico, a segunda hipótese é mais econômica, porém, do ponto de vista prático, não é possível saber o que ocorreu.

Monofilia de HuAscaromusca (Fig. 1)

\section{Condição autapomórfica:}

30 - fileira de cerdas discais no tergito 5 .

Utilizado por GuIMARÃEs (1977) como um dos estados de caráter para diagnose do táxon. Nenhuma outra espécie apresentou esta condição em Mesembrinellinae nem nos Calliphoridae examinados.

MonofiLia de EUMESEMBRINELla (Fig. 1)

GuIMARÃES (1977) fez a diagnose deste gênero utilizando estados primitivos de caráter com respeito ao táxon e estados derivados de caráter para pontos de análises internos. Apesar disto, a monofilia do gênero pode ser definida pelas seguintes autapomorfias:

31 - Fileiras de cerdas verticais do mero curvando-se levemente na porção superior (Fig. 2).

A presença de fileiras verticais no mero foi usada por MCALPINE (1989) como sinapomorfia na definição dos Oestroidea. Este caráter apresenta-se sob duas condições: a) fileiras verticais com quebras bruscas na porção superior, formando $\mathrm{L}$ invertido, ou b) levemente curvadas, assemelhando a arcos. Ambos os estados estão presentes dentro e fora de Mesembrinellinae. Neste táxon, somente Eumesembrinella apresenta a última condição. Considerando sua posição apical, advoga-se a interpretação de evolução independente para este estado.

32 - Dimorfismo sexual no padrão de coloração do abdome.

No macho, os tergitos abdominais $1+2$ e 3 apresentam a cor amarelolaranja como base, porém uma mancha de formato triangular ocorre na porção centro-posterior, podendo ou não se expandir lateralmente, formando uma faixa que geralmente não ultrapassa um terço do comprimento do tergito. Essa mancha é contígua ao tergito 4 , que é predominantemente escuro, a mesma coloração ocorre no tergito 5 . Na fêmea, os tergitos $1+2$ e 3 apresentam a coloração base semelhante a do macho, porém a mancha presente no tergito 3 é maior e sempre se expande lateralmente, ultrapassando um terço de seu comprimento; o tergito 4 
e o 5 são totalmente escuros (Fig. 2). Este dimorfismo no padrão de coloração do abdome é único com relação a Mesembrinellinae e considerado derivado.

Com relação ao parentesco filogenético das espécies de Eumesembrinella, nada foi proposto até o momento. MELLO (1967) e GuIMARÃES (1977) apresentaram apenas uma idéia de proximidade fenética entre $E$. quadrilineata e $E$. randa, quando da redescrição. Distinguindo estas espécies somente por um caráter, a presença e ausência de cerdas marginais no tergito 4 , respectivamente.

MONOFILIA DAS ESPÉCIES DE EUMESEMBRINELLA (Fig. 1)

\section{Eumesembrinella cyaneicincta}

\section{Estado autapomórfico:}

33 - Margem posterior dos tergitos 3 e 4 com uma distinta faixa violeta.

Este padrão de coloração ocorre apenas em E. cyaneicincta e $H$. aeneiventris. No entanto, a interpretação desta condição como evolução independente em ambas as linhagens é mais plausível do que a explicação de evolução única. Esta requer três hipóteses ad hoc de homoplasias, duas referentes às autapomorfias de Eumesembrinella e uma referente à reversão da estrutura autapomórfica de Huascaromusca.

\section{Eumesembrinella benoisti (Séguy, 1925), sp.rev.}

A presença de dois estados autapomórficos em $E$. benoisti indica-o como um grupo distinto de $E$. randa e demanda a revalidação da espécie. Estados autapomórficos:

34 - Ausência do padrão de mancha na parte distal da asa, próximo a veia Radiol e Radio2 +3 .

Este estado de caráter aparece nesta espécie e em $M$. bicolor. A interpretação por evolução independente desta condicão foi apresentada na discussão para $M$. bicolor. A justificativa para esta interpretação é fornecida pela grande distância filogenética existente entre as duas espécies.

35 - Fusionamento total do sintergito $7+8$ ao tergito 6 .

O fusionamento do sintergito $7+8$ ao tergito 6 foi assinalada como sinapomórfica para as espécies de Mesembrinella estudadas (exceto M. peregrina), Albuquerquea, Thompsoniella, Huascaramusca e Eumesembrinella. Porém, a fusão total ocorre somente em E. benoisti, condição considerada derivada.

A presença destes dois estados autapomórficos em $E$. benoisti indica-o como um grupo distinto de $E$. randa e demanda a revalidação de seu nome. 


\section{Eumesembrinella quadrilineata}

\section{Condição autapomórfica:}

36 - Ducto da espermateca com um estrangulamento próximo à base (Fig. 6)

Esta condição é única em Mesembrinellinae. Sendo considerada derivada para E. quadrilineata.

\section{Eumesembrinella randa}

\section{Condição autapomórfica:}

37 - Padrão de mancha na parte médio-apical da asa expandindo até a porção terminal da veia Subcosta.

Partindo dos Eumesembrinella, nenhuma espécie apresentou o padrão de mancha expandindo até a parte apical da veia subcosta, com exceção de $E$. randa, sendo este estado de caráter considerado autapomórfico.

MONOFILIA DE E. QUADRILINEATA (FABRICIUS, 1805) +E. RANDA (WALKER, 1849) + E. BENOISTI (SÉGUY, 1925) (Fig. 1)

Estas três espécies formam um agrupamento monofilético pelo compartilhamento das seguintes sinapomorfias:

38 - Região basal do esternito 5 do macho não arredondada (Fig. 10).

Em Mesembrinellinae, a região basal do esternito 5 do macho apresenta-se arredondada (Fig. 11). Em E. quadrilineata, E. randa e E. benoisti, a base deste esternito apresenta-se mais angulosa, sendo este estado considerado derivado.

39 - Ausência de mancha na porção distal do fềmur médio.

A presença de mancha na porção distal do fêmur foi utilizada como sinapomorfia na definição da monofilia de Thompsoniella, Huascaromusca e Eumesembrinella. Não obstante, esta condição está presente somente em $E$. cyaneicincta, no último táxon. Isto leva à hipótese de parentesco entre esta espécie e os dois primeiros gêneros, incompatível com as hipóteses anteriormente formuladas. Tomando o grupo Thompsoniella, Huascaromusca e Eumesembrinella, o estado de carater 24 (mancha distal no fêmur médio), que definia a monofilia deste agrupamento, agora é interpretado como sinapomórfico apenas para Thompsoniella, Huascaromusca e E. cyaneicincta. A sustentação desta hipótese como correta requer duas hipóteses ad hoc de homoplasia (os estados de caráter 31 e 32 que suportam a monofilia de Eumesembrinella). Por outro lado, a interpretação da ocorrência do padrão de mancha no fêmur como sinapomórfica para Thompsoniella, Huascaromusca e Eumesembrinella implica que sua ausência em $E$. cyaneicincta, E. quadrilineata e E. randa seja explicada como uma perda secundária (homoplásica com relação à ausência que ocorre fora do plano básico), sendo considerada derivada (sinapomórfica) para estas espécies (Fig. 1). A parcimônia neste caso leva a preferir a última hipótese.

Edeago com os ápices dos processos dorsolaterais com dentes (Fig. 16) 
(estado de caráter $15^{*}$ ) pode ser tomado como uma sinapomorfia para $E$. cyaneicincta $+E$. quadrilineata $+E$. randa. Visto que os ápices dos processos dorsolaterais na maioria dos Mesembrinellinae não apresentam dentes (Fig. 17), com exceção de Mesembrinella peregrina. Esta evidência conflita com as hipóteses anteriores. No entanto, a aceitação da monofilia entre $M$. peregrina e as demais espécies de Eumesembrinella implica necessariamente na exigência de mais hipóteses ad hoc de homoplasia do que uma interpretação por evolução independente desta condição em ambas as linhagens.

MONOFILIA dE E. RANDA E E. BENOISTIE A REJEIC̣Ão DA APROXIMAC̣Ão FENÉTICA ENTRE E. Quadrilineata E E. RANDA (Fig. 1)

\section{Estado de caráter sinapomórfico:}

40 - Ausência da fileira de cerdas margiais no tergito 4.

Este estado de caráter foi o único usado na separação de E. quadrilineata e E.randa. Deixando as duas espécies estreitamente relacionadas no que diz respeito a similaridade total. No entanto, a ausência da fileira de cerdas é única com respeito aos Mesembrinellinae, devendo ser tradada como derivada para $E$. randa e $E$. benoisti.

AGRADECIMENTOS. Aos curadores das coleções acima mencionadas pelo empréstimo do material. A D.S. Napp, A.P. do Prado e D.S. Amorim pela crítica do manuscrito. O autor senior agradece à K. Rognes pelas discussões e pela cessão de Helicobia palpalis. Ao Centro de Microscopia Eletrônica da Universidade Federal do Paraná, em especial à D.R. Eiras Stofella.

\section{REFERÊNCIAS BIBLIOGRÁFICAS}

ALDRICH, J.M. 1922. The Neotropical muscoid genus Mesembrinella Giglio-Tos and other testaceous muscoid flies. Proc. U.S. Nat. Mus. 62 (18): 1-36.

CRosskey, R.W. 1965. A systematic revision of the Ameniinae (Diptera, Calliphoridae). Bull. Brit. Mus. (Nat. Hist.) Entom. 16 (2): 35-140.

EldRedge, N. \& J. CRACRAFT. 1980. Phylogenetic patterns and the evolutionary process: Method and theory in comparative biology. New York, Columbia University Press, 349p.

GuimarÃEs, J.H. 1977. A systematic revision of the Mesembrinellidae, stat. nov.(Diptera, Cyclorrhapha). Archos. Zool., São Paulo, 29: 1-109.

Hall, D.G. 1948. The blowflies of North America. Mem. Entomol. Soc. Am. 4: $477 p$.

Hennig, W. 1965. Phylogenetic systematics. Ann. Rev. Ent. 10: 97-116.

1968. Elementos de uma sistemática filogenética. Buenos Aires, Editora Universitária de Buenos Aires, 353p.

1973. Ordnung Diptera (Zweiflügler). In: J.G. HELMCKE et al. (eds). Handbuch der Zoologie 4 (2): 337p.

Huckett, H.C \& J.R. VOCKeroth. 1987. Muscidae, p.675-1333. In: R.F. MCAlpine (ed). Manual of Neartic Diptera. Monograph no. 28. Otawa, 
Agriculture Canada, Research Branch, 2.

James, M.T. 1970. Família Calliphoridae. In: A catalogue of the Diptera of America South of the United States. São Paulo, Museu de Zoologia, Universidade de São Paulo, 102: 28p.

MCAlPINE, J.F. 1981. Morphology and Terminology. In: J.F. MCAlpine (ed.).

Manual of Neartic Diptera. Monograph 27. Otawa, Agricultulre Canada, Research Branch, 1: 167p.

1989. Phylogeny and classification of the Muscomorpha, p.1333-1581.

In: J.F. MCAlpine (ed.). Manual of Neartic Diptera. Monograph no. 32.

Otawa, Agriculture Canada, Research Branch, 3.

MCAlPine, J.F. \& D.D. MunRoE. 1968. Swarming of Loncheid flies and other insects, with descriptions of four new species of Lonchaeidae (Diptera). Can. Ent. 100: 1154-1178.

MELLO, R.P. DE. 1967. Contribuição aos Mesembrinellinae sul-americanos (Diptera, Calliphoridae). Studia Entom. 10: 1-80.

Nelson, G. \& N. PlatnicK. 1981. Systematics and Biogeography; cladistics and vicariance. New York, Columbia University Press, 567p.

PAPE, T. 1992. Phylogeny of the Tachinidae family-group (Diptera, Calyptrate). Tijdschr. Ent. 135: 43-86.

PERIS, S.V \& J.C. MARILuis. 1984. Notas sobre Mesembrinellidae. Eos. 60: 251-265.

Roback, S.S. 1951. A classification of the Muscoid Calyptrate Diptera. Ann. Entom. Soc. Am. 44 (3): 327-361.

Rognes, K. 1986. The systematic position of the genus Helicobosca Bezzi with a discussion of the monophyly of the calyptrate families Calliphoridae, Rhinophoridae, Sarcophagidae and Tachinidae (Diptera). Entomol. Scand. 17: 75-92.

SHANNON, R.C. 1923. Genera of neartic Calliphoridae (blowflies) with revision of Calliphorini (Diptera). Insect. Insc. Menstr. 11: 101-118.

SHEwEll, G.E. 1987. Calliphoridae, p.675-1333. In: J.F. MCAlPINE (ed). Manual of Neartic Diptera. Monograph no. 28. Otawa, Agriculture Canada, Research Branch. 2.

Townsend, C.H.T. 1931. Notes on American oestromuscoid types. Rev. Ent. 1: $65-104,157-183$.

. 1935. Manual of myiology. Itaquaquecetuba, 2.

WileY, O.E. 1981. Phylogenetics: The theory and practice of phylogenetic systematics. New York, John Willey and Sons, 439p. 